Research Article

\title{
Stability Analysis of Multistage One-Step Multiderivative Methods for Nonlinear Impulsive Differential Equations in Banach Spaces
}

\author{
Tao Long $\mathbb{D}$ and Yuexin Yu $\mathbb{D}$ \\ School of Mathematics and Computational Science, Xiangtan University, Xiangtan 411 105, China \\ Correspondence should be addressed to Yuexin Yu; yuyx@xtu.edu.cn
}

Received 8 August 2021; Revised 8 November 2021; Accepted 24 November 2021; Published 17 December 2021

Academic Editor: Saleh Mobayen

Copyright (c) 2021 Tao Long and Yuexin Yu. This is an open access article distributed under the Creative Commons Attribution License, which permits unrestricted use, distribution, and reproduction in any medium, provided the original work is properly cited.

\begin{abstract}
In this paper, we first introduce the problem class $K^{(p)}(\mu, \lambda, \zeta)$ with respect to the initial value problems of nonlinear impulsive differential equations in Banach spaces. The stability and asymptotic stability results of the analytic solution of the problem class $K^{(p)}(\mu, \lambda, \zeta)$ are obtained. Then, the numerical stability and asymptotic stability conditions of multistage one-step multiderivative methods are also given. Two numerical experiments are given to confirm the theoretical results in the end.
\end{abstract}

\section{Introduction}

Impulsive differential equations (IDEs) are widely applied in population control, infectious disease control, pest control, and control systems (see [1-4] and so on). Recently, the theory of impulsive differential equations has been widely studied by authors in [5-7] and so on. However, it is difficult to obtain the analytic solution of IDEs. Hence, taking numerical methods is a good choice. In [8], Wen et al. obtained the stability and asymptotic stability of the analytic solution and implicit Euler method for nonlinear IDEs in Banach spaces. The exponential stability of the numerical and analytical solutions of nonlinear IDEs is discussed by using a new technique in [9]. Zhang and Song studied the asymptotic stability of the analytic solution and Runge-Kutta methods for a class of piecewise continuous linear impulsive differential equations in [10]. The stability and asymptotic stability conditions of $(k, l)$-algebraically stable of Runge-Kutta methods for stiff impulsive differential equations have been studied by [11]. In [12], Mei proposed a new algorithm based on the reproducing kernel method and least square method for solving nonlinear IDEs. Zhang gave the asymptotic stability of the analytic solution of nonlinear impulsive differential equations under Lipschitz condition and studied the asymptotic stability of Runge-Kutta methods by using Pade approximation theory in [13].

Because of good stability and high accuracy, the multistage one-step multiderivative methods are widely used to solve ordinary differential equations (see [14-18]). In the present paper, we apply multistage one-step multiderivative methods to impulsive differential equations and analyze the stability and asymptotic stability of the methods in Banach spaces.

The rest of this paper is organized as follows. In Section 2 , the definition of the problem class $K^{(p)}(\mu, \lambda, \zeta)$ is given. In Section 3, for the problem class $K^{(p)}(\mu, \lambda, \zeta)$, we get the conditions of stability and asymptotic stability of the analytic solution. In Section 4, we obtain the stability and asymptotic stability of the multistage one-step multiderivative methods. In Section 5, we give relevant numerical experiments to verify the theoretical results. Finally, some conclusions are drafted in Section 6.

\section{Description of the Problem Class $K^{(p)}(\boldsymbol{\mu}, \lambda, \zeta)$}

Let $X$ be a real or complex Banach space with the norm $\|\cdot\|$, consider the following nonlinear IDEs: 


$$
\left\{\begin{array}{l}
y^{\prime}(t)=f(t, y(t)), \quad t>0, t \neq \tau_{k}, k=1,2, \ldots, \\
\left.\Delta y\right|_{t=\tau_{k}}=I_{k}\left(y\left(\tau_{k}\right)\right), \quad k=1,2, \ldots, \\
y\left(0^{+}\right)=y_{0},
\end{array}\right.
$$

where $\left.\Delta y\right|_{t=\tau_{k}}=y\left(\tau_{k}^{+}\right)-y\left(\tau_{k}\right), y\left(\tau_{k}^{+}\right)$denotes the right limit of $y(t)$ at $t=\tau_{k} ; I_{k}: X \longrightarrow X(k=1,2, \ldots$,$) are continuous$ mapping satisfying the following inequality:

$$
\begin{array}{r}
\left\|\eta_{1}+I_{k}\left(\eta_{1}\right)-\left(I_{k}\left(\eta_{2}\right)+\eta_{2}\right)\right\| \leq \gamma_{k}\left\|\eta_{1}-\eta_{2}\right\|, \\
\forall \eta_{1}, \eta_{2} \in X, k=1,2, \ldots,
\end{array}
$$

where $\gamma_{k}(k=1,2, \ldots$,$) are real constants with suitable size,$ and let $\zeta=\left(\gamma_{1}, \gamma_{2}, \ldots\right)$.

In this paper, we also assume that $f:(0,+\infty) \times X \longrightarrow X$ is a given continuous mapping and define a nonnegative function (cf., [14]):

$$
H_{u, v, t, f}(\xi)=\left\|u-v+\sum_{q=1}^{p}(-1)^{q} \xi_{q}\left[f^{(q-1)}(t, u)-f^{(q-1)}(t, v)\right]\right\|,
$$

where $\quad u, v \in X, t \geq 0, \xi=\left(\xi_{1}, \xi_{2}, \ldots, \xi_{p}\right)^{T} \in R^{p}\left(p \in N^{+}\right)$; the functions $f^{(q)}(t, x)$ are obtained by the following recurrence formula:

$$
\begin{aligned}
f^{(0)}(t, x) & =f(t, x), \\
f^{(q+1)}(t, x) & =\frac{\partial f^{(q)}(t, x)}{\partial t}+\frac{\partial f^{(q)}(t, x)}{\partial x} f(t, x) .
\end{aligned}
$$

For the convenience of illustration, we abbreviate $H_{u, v, t, f}(\xi)$ as $H(\xi)$. In addition, we define the following symbols:

$$
\begin{aligned}
& \qquad R_{-}^{n}:=\left\{\left(\alpha_{1}, \alpha_{2}, \ldots, \alpha_{n}\right)^{T} \in R^{n} \mid \quad \alpha_{i} \leq 0, i=1,2, \ldots, n\right\}, \\
& \alpha \leq \beta:=\left\{\forall \alpha=\left(\alpha_{1}, \alpha_{2}, \ldots, \alpha_{n}\right)^{T}, \beta=\left(\beta_{1}, \beta_{2}, \ldots, \beta_{n}\right)^{T} \in R^{n} \mid \quad \alpha_{i} \leq \beta_{i}, i=1,2, \ldots, n\right\} . \\
& \text { ose that } \mu, \lambda \in R_{-}^{p} \text {. The problem class }\left\{\begin{array}{l}
z^{\prime}(t)=f(t, z(t)), \quad t>0, t \neq \tau_{k}, k=1,2, \ldots, \\
\left.\Delta z\right|_{t=\tau_{k}}=I_{k}(z(t)), \quad k=1,2, \ldots, \\
z\left(0^{+}\right)=z_{0} .
\end{array}\right.
\end{aligned}
$$

Definition 1. Suppose that $\mu, \lambda \in R_{-}^{p}$. The problem class consisting of problem (1) is called the problem class $K^{(p)}(\mu, \lambda, \zeta)$ if the following inequality holds:

$$
H(\alpha) \leq \frac{1-\mu^{T}(\alpha-\lambda)}{1-\mu^{T}(\beta-\lambda)} H(\beta), \quad \forall \alpha, \beta \in R^{p}, \lambda \leq \alpha \leq \beta .
$$

Remark 1 . When $\zeta=0$, the initial value problem (1) degenerates to the initial value problem of ordinary differential equations. It can be seen that Definition 1 is a generalization of Definition 2.1 in [18].

Definition 2 (cf., [19]). The function $y:[0,+\infty) \longrightarrow X$ is said to be a solution of system (1) if the following conditions are satisfied:
(i) For $t>0, t \neq \tau_{k}, k=1,2, \ldots$, the function $y(t)$ is differentiable and $y^{\prime}(t)=f(t, y(t))$
(ii) The function $y(t)$ is left continuous in the interval $(0,+\infty)$ and $y\left(\tau_{k}^{+}\right)=y\left(\tau_{k}\right)+I_{k}\left(y\left(\tau_{k}\right)\right)$

(iii) $\lim _{t \longrightarrow 0^{+}} y(t)=y_{0}=y\left(0^{+}\right)$

\section{Stability Analysis with respect to the Problem Class $K^{(p)}(\boldsymbol{\mu}, \boldsymbol{\lambda}, \boldsymbol{\zeta})$}

In this section, we focus on the stability analysis of the analytical solution of the problem class $K^{(p)}(\mu, \lambda, \zeta)$. First, we introduce the perturbed problem of (1):
Lemma 1 (cf., [18]). If the initial value problem (1) belongs to the problem class $K^{(p)}(\mu, \lambda, \zeta)$, then

$$
\begin{array}{r}
\left\|y\left(t_{2}\right)-z\left(t_{2}\right)\right\| \leq e^{\left(\mu_{1} /\left(1+\mu^{T} \lambda\right)\right)\left(t_{2}-t_{1}\right)}\left\|y\left(t_{1}\right)-z\left(t_{1}\right)\right\|, \\
\forall \tau_{k-1}<t_{1} \leq t_{2} \leq \tau_{k},(k=1,2, \ldots),
\end{array}
$$

where $\mu_{1}, z(t)$ represent the first component of $\mu$ and the solution of the perturbed problem (7), respectively.

Theorem 1. If the initial value problem (1) belongs to the problem class $K^{(p)}(\mu, \lambda, \zeta)$, then for $\forall t \in\left(\tau_{k-1}\right.$, $\left.\tau_{k}\right](k=1,2, \ldots)$, we have

$$
\begin{aligned}
\|y(t)-z(t)\| \leq & e^{\left(\mu_{1} /\left(1+\mu^{T} \lambda\right)\right)\left(t-\tau_{k-1}\right)} \prod_{i=1}^{k-1} \gamma_{i} \\
& e^{\left(\mu_{1} /\left(1+\mu^{T} \lambda\right)\right)\left(\tau_{i}-\tau_{i-1}\right)}\left\|y_{0}-z_{0}\right\| .
\end{aligned}
$$

Inequality (9) characterizes the stability of the initial value problem (1).

Proof. Since $y_{0}=y\left(0^{+}\right)$and $z_{0}=z\left(0^{+}\right)$, then by Lemma 1, for $\forall t \in\left(0, \tau_{1}\right)$, we have

$$
\|y(t)-z(t)\| \leq e^{\left(\mu_{1} /\left(1+\mu^{T} \lambda\right)\right)\left(t-0^{+}\right)}\left\|y_{0}-z_{0}\right\| .
$$

On the other hand, from (2), we get 


$$
\begin{aligned}
\left\|y\left(\tau_{k}^{+}\right)-z\left(\tau_{k}^{+}\right)\right\| & =\left\|y\left(\tau_{k}\right)+I_{k}\left(y\left(\tau_{k}\right)\right)-z\left(\tau_{k}\right)-I_{k}\left(z\left(\tau_{k}\right)\right)\right\| \\
& \leq \gamma_{k}\left\|y\left(\tau_{k}\right)-z\left(\tau_{k}\right)\right\| \\
& \leq \gamma_{k} e^{\left(\mu_{1} /\left(1+\mu^{T} \lambda\right)\right)\left(\tau_{k}-\tau_{k-1}\right)}\left\|y\left(\tau_{k-1}^{+}\right)-z\left(\tau_{k-1}^{+}\right)\right\|, \quad(k=1,2, \ldots) .
\end{aligned}
$$

By induction, when $t \in\left(\tau_{k-1}, \tau_{k}\right](k=1,2, \ldots)$, we have

$$
\begin{aligned}
\|y(t)-z(t)\| & \leq e^{\left(\mu_{1} /\left(1+\mu^{T} \lambda\right)\right)\left(t-\tau_{k-1}\right)} \gamma_{k-1} e^{\left(\mu_{1} /\left(1+\mu^{T} \lambda\right)\right)\left(\tau_{k-1}-\tau_{k-2}\right)}\left\|y\left(\tau_{k-2}^{+}\right)-z\left(\tau_{k-2}^{+}\right)\right\| \\
& \leq e^{\left(\mu_{1} /\left(1+\mu^{T} \lambda\right)\right)\left(t-\tau_{k-1}\right)} \prod_{i=1}^{k-1} \gamma_{i} e^{\left(\mu_{1} /\left(1+\mu^{T} \lambda\right)\right)\left(\tau_{i}-\tau_{i-1}\right)}\left\|y_{0}-z_{0}\right\| .
\end{aligned}
$$

This completes the proof of Theorem 1.

It is easy to get the following corollary from Theorem 1.

Corollary 1. If the initial value problem (1) belongs to the problem class $K^{(p)}(\mu, \lambda, \zeta)$, there is a constant $0<\delta<1$ such that

$$
\gamma_{i} e^{\left(\mu_{1} /\left(1+\mu^{T} \lambda\right)\right)\left(\tau_{i}-\tau_{i-1}\right)} \leq \delta, \quad i=1,2, \ldots,
$$

then

$$
\lim _{t \longrightarrow+\infty}\|y(t)-z(t)\|=0 .
$$

The above relation characterizes the asymptotic stability of the initial value problem (1).

\section{Stability and Asymptotic Stability of \\ Multistage One-Step \\ Multiderivative Methods}

The multistage one-step multiderivative methods for solving ordinary differential equations

$$
\left\{\begin{array}{l}
y^{\prime}(t)=f(t, y(t)), \quad t>0, \\
y(0)=y_{0},
\end{array}\right.
$$

can be expressed as follows (cf., [18]):

$$
\left\{\begin{array}{l}
Y_{i}=y_{l}+\sum_{q=1}^{p}(-1)^{q-1} h^{q} \sum_{j=1}^{i} a_{i j}^{(q)} f^{(q-1)}\left(t_{l}+c_{j} h, Y_{j}\right), \quad i=1,2, \ldots, s, \\
y_{l+1}=y_{l}+\sum_{q=1}^{p}(-1)^{q-1} h^{q} \sum_{j=1}^{s} b_{j}^{(q)} f^{(q-1)}\left(t_{l}+c_{j} h, Y_{j}\right) .
\end{array}\right.
$$

Applying method (16) for solving problem (1), we have

$$
\begin{cases}Y_{i}^{k, l}=y_{k, l-1}+\sum_{q=1}^{p}(-1)^{q-1} h_{k}^{q} \sum_{j=1}^{i} a_{i j}^{(q)} f^{(q-1)}\left(t_{k, l-1}+c_{j} h_{k}, Y_{j}^{k, l}\right), & i=1,2, \ldots, s, \\ y_{k, l}=y_{k, l-1}+\sum_{q=1}^{p}(-1)^{q-1} h_{k}^{q} \sum_{j=1}^{s} b_{j}^{(q)} f^{(q-1)}\left(t_{k, l-1}+c_{j} h_{k}, Y_{j}^{k, l}\right), & l=1,2, \ldots, m, \\ y_{k+1,0}=y_{k, m}+I_{k+1}\left(y_{k, m}\right), & k=1,2, \ldots, n, \\ y_{0,0}=y_{0}, & \end{cases}
$$

where the parameters $a_{i j}^{(q)}$ and $b_{j}^{(q)}$ are real constants, $0 \leq c_{j} \leq 1, h_{k}=\left(\left(\tau_{k+1}-\tau_{k}\right) / m\right)(m \geq 1)$ is a given step-size in $\left(\tau_{k}, \tau_{k+1}\right], t_{k, l-1}=\tau_{k}+(l-1) h_{k}$, and $y_{k, l}, y_{k+1,0}$, and $Y_{j}^{k, l}$ are approximations to the exact solution $y\left(t_{k, l}\right), y\left(t_{k+1,0}^{+}\right)$, and $y\left(t_{k, l-1}+c_{j} h_{k}\right)$, respectively. In the present paper, we always assume that $a_{i i}^{(q)} \geq 0(i=1, \ldots, s, q=1, \ldots, p)$. 
Remark 2. If there exists at least one $i$ such that $a_{i i}^{(q)}>0$, then method (17) is implicit; otherwise, it is explicit; if $p=1$, method (17) is the explicit or diagonally implicit Runge-Kutta methods.
Similarly, applying the same methods (16) to the perturbed problem (7) yields

$$
\begin{cases}Z_{i}^{k, l}=z_{k, l-1}+\sum_{q=1}^{p}(-1)^{q-1} h_{k}^{q} \sum_{j=1}^{i} a_{i j}^{(q)} f^{(q-1)}\left(t_{k, l-1}+c_{j} h_{k}, Z_{j}^{k, l}\right), & i=1,2, \ldots, s, \\ z_{k, l}=z_{k, l-1}+\sum_{q=1}^{p}(-1)^{q-1} h_{k}^{q} \sum_{j=1}^{s} b_{j}^{(q)} f^{(q-1)}\left(t_{k, l-1}+c_{j} h_{k}, Z_{j}^{k, l}\right), & l=1,2, \ldots, m, \\ z_{k+1,0}=z_{k, m}+I_{k+1}\left(z_{k, m}\right), & k=1,2, \ldots, n, \\ z_{0,0}=z_{0} . & \end{cases}
$$

For the convenience of the following analysis, we introduce the following symbols:

$$
\left\{\begin{array}{l}
W_{i}^{k, l}=Y_{i}^{k, l}-Z_{i}^{k, l}, \quad i=1,2, \ldots, s \\
Q_{j, k, l}^{(q)}=h_{k}^{q}\left[f^{(q-1)}\left(t_{k, l-1}+c_{j} h_{k}, Y_{j}^{k, l}\right)-f^{(q-1)}\left(t_{k, l-1}+c_{j} h_{k}, Z_{j}^{k, l}\right)\right] \\
w_{k, l}=y_{k, l}-z_{k, l} \\
a_{s+1, j}^{(q)}=b_{j}^{(q)} \\
Q_{s+1, k, l}^{(q)}=a_{s+1, s+1}^{(q)}=0, j=1,2, \ldots, i, q=1,2, \ldots, p
\end{array}\right.
$$

Then, a combination of (17) and (18) leads directly to

$$
\left\{\begin{array}{l}
W_{i}^{k, l}=w_{k, l-1}+\sum_{q=1}^{p}(-1)^{q-1} \sum_{j=1}^{i} a_{i j}^{(q)} Q_{j, k, l}^{(q)}, \quad i=1,2, \ldots, s, \\
w_{k, l}=w_{k, l-1}+\sum_{q=1}^{p}(-1)^{q-1} \sum_{j=1}^{s} b_{j}^{(q)} Q_{j, k, l}^{(q)}, \quad l=1,2, \ldots, m, k=1,2, \ldots, n, \\
w_{k+1,0}=\varphi_{k+1}\left(y_{k, m}\right)-\varphi_{k+1}\left(z_{k, m}\right),
\end{array}\right.
$$

where $\varphi_{k+1}\left(y_{k, m}\right)=y_{k, m}+I_{k+1}\left(y_{k, m}\right)$.

Now, we give a set of $s \times s$ lower triangular matrix $D^{(q)}=$ $\left(d_{i j}^{(q)}\right)(q=1,2, \ldots, p)$ and construct a set of $s \times s$ lower triangular matrix $G^{(q)}=\left(g_{i j}^{(q)}\right)(q=1,2, \ldots, p)$ by matrix group $D^{(q)}$ and method (17), where

$$
g_{i j}^{(q)}= \begin{cases}0, & \sum_{\tilde{q}=1}^{p} d_{i j}^{(\widetilde{q})}=0, \sum_{\bar{q}=1}^{p} \sum_{k=j}^{i}\left(d_{i k}^{(\bar{q})} a_{k j}^{(q)}-a_{i+1, j}^{(q)}\right)=0, \\ +\infty, & \sum_{\tilde{q}=1}^{p} d_{i j}^{(\widetilde{q})}=0, \sum_{\bar{q}=1}^{p} \sum_{k=j}^{i}\left(d_{i k}^{(\bar{q})} a_{k j}^{(q)}-a_{i+1, j}^{(q)}\right) \neq 0, \\ \frac{\sum_{\bar{q}=1}^{p} \sum_{k=j}^{i}\left(d_{i k}^{(\bar{q})} a_{k j}^{(q)}-a_{i+1, j}^{(q)}\right)}{\sum_{\tilde{q}=1}^{p} d_{i j}^{(\widetilde{q})}}, & \sum_{\widetilde{q}=1}^{p} d_{i j}^{(\widetilde{q})} \neq 0, j=1,2, \ldots, i, i=1,2, \ldots, s .\end{cases}
$$


Definition 3 (cf., [18]). If the elements of the matrix group $D^{(q)}=\left(d_{i j}^{(q)}\right)(q=1,2, \ldots, p)$ satisfying

$$
g_{i j}^{(q)} \leq a_{j j}^{(q)}, \quad j=1,2, \ldots, i, i=1,2, \ldots, s, q=1,2, \ldots, p,
$$

then it is said that the matrix group $D^{(q)}(q=1,2, \ldots, p)$ and method (17) is $G_{p}-$ consistent.

Definition 4 (cf., [18]). If a set of nonnegative $s \times s$ lower triangular matrix $D^{(q)}(q=1,2, \ldots, p)$ is $G_{p}$ - consistent, and $D^{(q)}$ satisfies $\max _{1 \leq q \leq p}\left\|D^{(q)}\right\|_{\infty} \leq(1 / p)$, then method (17) is said to be $G_{p}$-stable with respect to $D^{(q)}(q=1,2, \ldots, p)$.

Theorem 2. Suppose that method (17) is $G_{p}-$ stable. Then, the numerical solutions $y_{k, l}$ and $z_{k, l}$, obtained by using method (17) to problems (1) and (7) which belong to the problem class $K^{(p)}(\mu, \lambda, \zeta)$, respectively, satisfy the stability inequality

$$
\begin{array}{r}
\left\|y_{k, l}-z_{k, l}\right\| \leq \phi^{l}\left(h_{k}\right) \prod_{i=1}^{k} \phi^{m}\left(h_{i-1}\right) \gamma_{i}\left\|y_{0}-z_{0}\right\|, \\
l=1,2, \ldots, m, \forall h_{k} \in(0, H] \backslash+\infty
\end{array}
$$

where

$$
\begin{aligned}
H & =\min _{1 \leq q \leq p}\left\{\min _{1 \leq i \leq s}\left[\min _{1 \leq j \leq i} h_{i j}^{(q)}\right]\right\}, \\
h_{i j}^{(q)} & = \begin{cases}\left(\frac{\lambda_{q}}{g_{i j}^{(q)}}\right)^{1 / q}, & g_{i j}^{(q)}<0, \\
+\infty, & g_{i j}^{(q)} \geq 0 .\end{cases}
\end{aligned}
$$

$\phi\left(h_{k}\right)$ is a positive, continuous, and nonincreasing function in the interval $(0, H]$, and it can be calculated by the following recurrence formula:

$$
\left\{\begin{array}{l}
\phi_{1}\left(h_{k}\right)=1, \\
\phi_{i+1}\left(h_{k}\right)=\left|1-\sum_{q=1}^{p} \sum_{j=1}^{i} d_{i j}^{(q)}\right|+\sum_{q=1}^{p} \sum_{j=1}^{i}\left|d_{i j}^{(q)}\right| \chi_{i j}\left(h_{k}\right) \phi_{j}\left(h_{k}\right), \\
\phi\left(h_{k}\right)=\phi_{s+1}\left(h_{k}\right), \\
\chi_{i j}\left(h_{k}\right)=\frac{1-\mu^{T}\left(g_{i j}^{h_{k}}-\lambda\right)}{1-\mu^{T}\left(a_{j j}^{h_{k}}-\lambda\right)}, \quad i=1,2, \ldots, s, j=1,2, \ldots, i,
\end{array}\right.
$$

where $g_{i j}^{h_{k}}=\left(h_{k} g_{i j}^{(1)}, \ldots, h_{k}^{p} g_{i j}^{(p)}\right)^{T} \in R^{p}(i, j=1, \ldots, s), a_{j j}^{h_{k}}$ $=\left(h_{k} a_{j j}^{(1)}, \ldots, h_{k}^{p} a_{j j}^{(p)}\right)^{T} \in R^{p}(j=1,2, \ldots, i, i=1,2, \ldots, s)$.

Proof. For $\forall h_{k} \in(0, H] \backslash+\infty$, from (20), we have

$$
W_{i}^{k, l}-\sum_{q=1}^{p}(-1)^{q-1} a_{i i}^{(q)} Q_{i, k, l}^{(q)}=w_{k, l-1}+\sum_{q=1}^{p}(-1)^{q-1} \sum_{j=1}^{i-1} a_{i j}^{(q)} Q_{j, k, l}^{(q)} \text {, }
$$

where $i=1,2, \ldots, s+1$.

Next, we shall prove the following inequality by mathematical induction:

$$
\begin{aligned}
\left\|W_{i}^{k, l}-\sum_{q=1}^{p}(-1)^{q-1} a_{i i}^{(q)} Q_{i, k, l}^{(q)}\right\| & \leq \phi_{i}\left(h_{k}\right)\left\|w_{k, l-1}\right\|, \\
i & =1,2, \ldots, s+1 .
\end{aligned}
$$

When $i=1$, by (26), we can easily get

$$
\left\|W_{1}^{k, l}-\sum_{q=1}^{p}(-1)^{q-1} a_{11}^{(q)} Q_{1, k, l}^{(q)}\right\| \leq \phi_{1}\left(h_{k}\right)\left\|w_{k, l-1}\right\| .
$$
holds:

If there exists $i=\widetilde{k}$, such that the following inequality

$$
\left\|W_{\widetilde{k}}^{k, l}-\sum_{q=1}^{p}(-1)^{q-1} \underset{k k}{a}{\underset{\widetilde{k}}{(q)}(q),}_{(q)}^{(q)}\right\| \leq \phi_{\vec{k}}^{-}\left(h_{k}\right)\left\|w_{k, l-1}\right\|,
$$

then for $i=\widetilde{k}+1$, by (26), we have 


$$
\begin{aligned}
& W_{\tilde{k}+1}^{k, l}-\sum_{q=1}^{p}(-1)^{q-1} \underset{k+1, \tilde{k}+1}{a_{k+1, k, l}^{(q)}} Q_{\tilde{k}^{(q)}}^{p} \\
& =w_{k, l-1}+\sum_{q=1}^{p}(-1)^{q-1} \sum_{i=1}^{\tilde{k}} \underset{k+1, i}{a_{i, k, l}^{(q)}} Q^{(q)} \\
& =\left(1-\sum_{q=1}^{p} \sum_{i=1}^{\tilde{k}} d_{k i}^{(q)}\right) w_{k, l-1}+\sum_{q=1}^{p}(-1)^{q-1} \sum_{i=1}^{n} a_{k+1, i}^{(q)} Q_{i, k, l}^{(q)}+\sum_{q=1}^{p} \sum_{i=1}^{\tilde{k}} d_{k i}^{(q)} w_{k, l-1} .
\end{aligned}
$$

From (20), we can get

$$
w_{k, l-1}=W_{i}^{k, l}-\sum_{q=1}^{p}(-1)^{q-1} \sum_{j=1}^{i} a_{i j}^{(q)} Q_{j, k, l}^{(q)} .
$$

$$
\begin{aligned}
& W_{\widetilde{k}+1}^{k, l}-\sum_{q=1}^{p}(-1)^{q-1} a_{k+1, \tilde{k}+1}^{(q)} Q_{\tilde{k}^{(q)}, k, l}^{(q)} \\
& =\left(1-\sum_{q=1}^{p} \sum_{i=1}^{\tilde{k}} d_{k i}^{(q)}\right) w_{k, l-1}+\sum_{q=1}^{p}(-1)^{q-1} \sum_{i=1}^{\tilde{k}} a_{k+1, i}^{(q)} Q_{i, k, l}^{(q)} \\
& +\sum_{q=1}^{p} \sum_{i=1}^{\tilde{k}} d_{k i}^{(q)}\left(W_{i}^{k, l}-\sum_{\bar{q}=1}^{p}(-1)^{\bar{q}-1} \sum_{j=1}^{i} a_{i j}^{(\bar{q})} Q_{j, k, l}^{(\bar{q})}\right) \\
& =\left(1-\sum_{q=1}^{p} \sum_{i=1}^{\tilde{k}} d_{k i}^{(q)}\right) w_{k, l-1}+\sum_{q=1}^{p} \sum_{i=1}^{\tilde{k}}\left[(-1)^{q-1} \underset{k+1, i}{a_{i, k, l}^{(q)}} Q_{k i}^{(q)}+d_{i}^{(q)} W_{i}^{k, l}\right] \\
& -\sum_{q=1}^{p} \sum_{\bar{q}=1}^{p} \sum_{j=1}^{\tilde{k}} \sum_{i=j}^{\tilde{k}}(-1)^{\bar{q}-1} d_{k i}^{(q)} a_{i j}^{(\bar{q})} Q_{j, k, l}^{(\bar{q})} \\
& =\left(1-\sum_{q=1}^{p} \sum_{i=1}^{\tilde{k}} d_{k i}^{(q)}\right) w_{k, l-1}+\sum_{\tilde{q}=1}^{p} \sum_{i=1}^{\tilde{k}} d_{k i}^{(\tilde{q})} W_{i}^{k, l}
\end{aligned}
$$

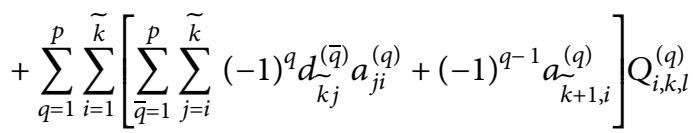

$$
\begin{aligned}
& =\left(1-\sum_{q=1}^{p} \sum_{i=1}^{\tilde{k}} d_{k i}^{(q)}\right) w_{k, l-1} \\
& +\sum_{i=1}^{\tilde{k}}\left\{\sum_{\widetilde{q}=1}^{p} d_{k i}^{(\widetilde{q})}\left[W_{i}^{k, l}+\frac{\sum_{q=1}^{p}(-1)^{q}\left(\sum_{\bar{q}=1}^{p} \sum_{j=i}^{\tilde{k}} d_{k j}^{(\bar{q})} a_{j i}^{(q)}-a_{k+1, i}^{(q)}\right) Q_{i, k, l}^{(q)}}{\sum_{\tilde{q}=1}^{p} d_{k i}^{(\widetilde{q})}}\right]\right\} \\
& =\left(1-\sum_{q=1}^{p} \sum_{i=1}^{\tilde{k}} d_{k i}^{(q)}\right) w_{k, l-1}+\sum_{i=1}^{\tilde{k}} \sum_{\tilde{q}=1}^{p} d_{k i}^{(\widetilde{q})}\left[W_{i}^{k, l}+\sum_{q=1}^{p}(-1)^{q} g_{k i}^{(q)} Q_{i, k, l}^{(q)}\right],
\end{aligned}
$$


where $g_{\sim_{k}}^{(q)}$ can be calculated by (21). Then, a combination of (22) and (6) leads directly to

$$
H_{j}\left(g_{i j}^{h_{k}}\right) \leq \chi_{i j}\left(h_{k}\right) H_{j}\left(a_{j j}^{h_{k}}\right), \quad j=1,2, \ldots, i ; i=1,2, \ldots, s .
$$

By (29), we obtain

$$
\begin{aligned}
H_{j}\left(a_{j j}^{h_{k}}\right)= & \| Y_{j}^{k, l}-Z_{j}^{k, l}+\sum_{q=1}^{p}(-1)^{q} h_{k}^{q} a_{j j}^{(q)}\left[f^{(q-1)}\left(t_{k, l-1}+c_{j} h_{k}, Y_{j}^{k, l}\right)\right. \\
& \left.-f^{(q-1)}\left(t_{k, l-1}+c_{j} h_{k}, Z_{j}^{k, l}\right)\right] \| \\
= & \left\|W_{j}^{k, l}+\sum_{q=1}^{p}(-1)^{q} a_{j j}^{(q)} Q_{j, k, l}^{(q)}\right\| \\
\leq & \phi_{j}\left(h_{k}\right)\left\|w_{k, l-1}\right\| .
\end{aligned}
$$

From equations (33) and (34) and through the induction method, we can obtain

$$
\begin{aligned}
& \left\|W_{\widetilde{k}+1}^{k, l}-\sum_{q=1}^{p}(-1)^{q-1} \underset{k+1, k+1}{a_{(q)}^{(q)}} Q_{\tilde{k}^{(q)}, k, l}^{(q)}\right\| \\
& \leq\left|1-\sum_{q=1}^{p} \sum_{i=1}^{\tilde{k}} d_{k i}^{(q)}\right|\left\|w_{k, l-1}\right\|+\sum_{i=1}^{\tilde{k}} \sum_{\tilde{q}=1}^{p}\left|d_{k i}^{(\widetilde{q})}\right| H_{i}\left(g_{k i}^{h_{k}}\right) \\
& \leq\left|1-\sum_{q=1}^{p} \sum_{i=1}^{\tilde{k}} d_{k i}^{(q)}\right|\left\|w_{k, l-1}\right\|+\sum_{i=1}^{\tilde{k}} \sum_{\tilde{q}=1}^{p}\left|d_{k i}^{(\widetilde{q})}\right| \chi_{k i}\left(h_{k}\right) H_{i}\left(a_{i i}^{h_{k}}\right) \\
& \leq\left[\left|1-\sum_{q=1}^{p} \sum_{i=1}^{\tilde{k}} \underset{k i}{d^{(q)}}\right|+\sum_{i=1}^{\tilde{k}} \sum_{\tilde{q}=1}^{p}\left|\underset{k i}{d_{(\tilde{q})}^{(\tilde{q})}}\right| \chi_{k i}\left(h_{k}\right) \phi_{i}\left(h_{k}\right)\right]\left\|w_{k, l-1}\right\| \\
& =\phi_{\widetilde{k}^{-1}}\left(h_{k}\right)\left\|w_{k, l-1}\right\| \text {. }
\end{aligned}
$$

For (26), let $i=s+1$, then we have

$$
\left\|w_{k, l}\right\|=\left\|W_{s+1}^{k, l}-\sum_{q=1}^{p}(-1)^{q-1} a_{s+1, s+1}^{(q)} Q_{s+1, k, l}^{(q)}\right\| \leq \phi\left(h_{k}\right)\left\|w_{k, l-1}\right\|,
$$

which implies that

$$
\left\|y_{k, l}-z_{k, l}\right\| \leq \phi\left(h_{k}\right)\left\|y_{k, l-1}-z_{k, l-1}\right\| .
$$

Note that

$$
\left\|y_{k, 0}-z_{k, 0}\right\| \leq \gamma_{k}\left\|y_{k-1, m}-z_{k-1, m}\right\| .
$$

Therefore, we obtain

$$
\begin{aligned}
\left\|y_{k, l}-z_{k, l}\right\| & \leq \phi^{l}\left(h_{k}\right)\left\|y_{k, 0}-z_{k, 0}\right\| \\
& \leq \phi^{l}\left(h_{k}\right) \gamma_{k}\left\|y_{k-1, m}-z_{k-1, m}\right\| \\
& \leq \phi^{l}\left(h_{k}\right) \prod_{i=1}^{k} \phi^{m}\left(h_{i-1}\right) \gamma_{i}\left\|y_{0}-z_{0}\right\| .
\end{aligned}
$$

This completes the proof of Theorem 2 .

From Theorem 2, the following corollary can be obtained.

Corollary 2. If there is a real constant $0<\rho<1$ such that

$$
\phi^{m}\left(h_{i-1}\right) \gamma_{i} \leq \rho, \quad i=1,2, \ldots,
$$

and the conditions in Theorem 2 hold, then

$$
\lim _{k \longrightarrow+\infty}\left\|y_{k, l}-z_{k, l}\right\|=0 \text {. }
$$

The above formula shows that method (17) is asymptotically stable when solving the problem class $K^{(p)}(\mu, \lambda, \zeta)$.

Remark 3. Most of the literature on the stability analysis of the numerical method for impulsive differential equations is based on the classic Lipschitz or one-sided Lipschitz conditions in the sense of the standard inner product norm (see $[9,13,20])$. If the value of the one-sided Lipschitz constant of the problem is very large (see problem (42)), these classic stability theories will fail. Therefore, in order to overcome the standard inner product norm, we analyze the stability in Banach space. So, the research in this paper has certain value.

\section{Numerical Experiments}

Example 1. Consider the following nonlinear IDEs:

$$
\left\{\begin{array}{l}
y^{\prime}(t)=\left(\begin{array}{cc}
-2 & 10^{7} \\
0 & -2-10^{7}
\end{array}\right) y(t)+\left(\begin{array}{c}
\sin \left(y_{2}(t)\right) \\
-\sin \left(y_{2}(t)\right)
\end{array}\right), \quad 0<t, t \neq k, k=1,2, \ldots \\
\Delta y(t)=\left(\begin{array}{ll}
0.6 & 0.6 \\
0.4 & 0.5
\end{array}\right) y(t), \quad t=k \\
y\left(0^{+}\right)=(0.5,0.5)^{T} .
\end{array}\right.
$$

The corresponding perturbed problem is 


$$
\left\{\begin{array}{l}
z^{\prime}(t)=\left(\begin{array}{cc}
-2 & 10^{7} \\
0 & -2-10^{7}
\end{array}\right) z(t)+\left(\begin{array}{c}
\sin \left(z_{2}(t)\right) \\
-\sin \left(z_{2}(t)\right)
\end{array}\right), \quad 0<t, t \neq k, k=1,2, \ldots, \\
\Delta z(t)=\left(\begin{array}{cc}
0.6 & 0.6 \\
0.4 & 0.5
\end{array}\right) y(t), \quad t=k, \\
z\left(0^{+}\right)=(1,1)^{T} .
\end{array}\right.
$$

The Jacobi matrix of the right-side function $f(y(t))$ of problem

is $J=(\partial f / \partial y)=$ $\left(\begin{array}{cc}-2 & 10^{7}+\cos \left(y_{2}(t)\right) \\ 0 & -2-10^{7}-\cos \left(y_{2}(t)\right)\end{array}\right)$. In the standard inner product space, the logarithmic matrix norm is $m=((\sqrt{2}-1) / 2) \times\left(10^{7}+\cos \left(y_{2}(t)\right)-2\right.$. That is to say, the one-sided Lipschitz constant is a huge positive value, so the classical stability theory fails. However, under the 1 norm, it is easy to know that problem (42) belongs to the problem class $K^{(p)}(\mu, \lambda, \zeta)$, where $p=2, \mu=(-2,-4)^{T}, \lambda=$ $(-(1 / 2),-(1 / 2))^{T}$, and $\zeta=(1.1, \ldots, 1.1)^{T}$.

We consider the third-order Obrechkoff method as follows:

$$
\left\{\begin{array}{l}
Y_{1}^{k}=y_{k, l-1}, \\
Y_{2}^{k}=y_{k, l-1}+h_{k}\left[\frac{1}{3} f\left(t_{k, l-1}, Y_{1}^{k}\right)+\frac{2}{3} f\left(t_{k, l}, Y_{2}^{k}\right)\right]-\frac{h^{2}}{6} f^{\prime}\left(t_{k, l}, Y_{2}^{k}\right) \\
y_{k, l}=y_{k, l-1}+h_{k}\left[\frac{1}{3} f\left(t_{k, l-1}, Y_{1}^{k}\right)+\frac{2}{3} f\left(t_{k, l}, Y_{2}^{k}\right)\right]-\frac{h^{2}}{6} f^{\prime}\left(t_{k, l}, Y_{2}^{k}\right) .
\end{array}\right.
$$

Take

$$
\begin{aligned}
& D^{(1)}=\left(\begin{array}{cc}
\frac{1}{3} & 0 \\
\frac{1}{4} & \frac{1}{4}
\end{array}\right), \\
& D^{(2)}=\left(\begin{array}{cc}
\frac{1}{3} & 0 \\
\frac{1}{3} & \frac{1}{6}
\end{array}\right),
\end{aligned}
$$

$$
\begin{aligned}
& G^{(1)}=\left(\begin{array}{rr}
-\frac{1}{2} & 0 \\
1 & 14 \\
-\frac{1}{3} & -\frac{15}{15}
\end{array}\right), \\
& G^{(2)}=\left(\begin{array}{cc}
0 & 0 \\
0 & -\frac{7}{30}
\end{array}\right) .
\end{aligned}
$$

So, we can verify that method (44) is $G_{p}-$ stable. For $\forall h_{k} \in(0, H]$, when method (44) is applied to problems (42) and (43), we have the following stability inequalities: 


$$
\begin{array}{r}
\left\|y_{k, l}-z_{k, l}\right\| \leq 2.1 \times \phi^{l}\left(h_{k}\right) \prod_{i=1}^{k} \phi^{m}\left(h_{i-1}\right)\left\|y_{0}-z_{0}\right\|, \\
l=1,2, \ldots, m,
\end{array}
$$

where $H=\min \left\{-(15 / 14) \lambda_{1}, \sqrt{-(30 / 7) \lambda_{2}}\right\}=(15 / 28), \phi\left(h_{k}\right)$ can be obtained by (25).
According to Corollary 2, when we take $h_{k}=(1 / 10)$, the third-order Obrechkoff method (44) for (42) is asymptotically stable (see Figure 1).

Example 2. Consider the following nonlinear IDEs:

$$
\left\{\begin{array}{l}
y^{\prime}(t)=\left(\begin{array}{cc}
-3 & 10 \\
0 & -13
\end{array}\right) y(t)+\left(\begin{array}{c}
\cos \left(y_{2}(t)\right) \\
-\cos \left(y_{2}(t)\right)
\end{array}\right), \quad 0<t, t \neq k, k=1,2, \ldots \\
\Delta y(t)=\left(\begin{array}{ll}
0.7 & 0.5 \\
0.3 & 0.5
\end{array}\right) y(t), \quad t=k \\
y\left(0^{+}\right)=(0,0)^{T}
\end{array}\right.
$$

The corresponding perturbed problem is

$$
\left\{\begin{array}{l}
z^{\prime}(t)=\left(\begin{array}{cc}
-3 & 10 \\
0 & -13
\end{array}\right) z(t)+\left(\begin{array}{c}
\cos \left(z_{2}(t)\right) \\
-\cos \left(z_{2}(t)\right)
\end{array}\right), \quad 0<t, t \neq k, k=1,2, \ldots \\
\Delta z(t)=\left(\begin{array}{ll}
0.7 & 0.5 \\
0.3 & 0.5
\end{array}\right) z(t), \quad t=k \\
z\left(0^{+}\right)=(1,1)^{T}
\end{array}\right.
$$

The Jacobi matrix of the right-side function $f(y(t))$ of problem (48) is $J=(\partial f / \partial y)=\left(\begin{array}{cc}-3 & 10-\sin \left(y_{2}(t)\right) \\ 0 & -13+\sin \left(y_{2}(t)\right)\end{array}\right)$. Under the 1 norm, the logarithmic matrix norm is $m=-3$.
So, it is easy to know that problem (48) belongs to the problem class $K^{(p)}(\mu, \lambda, \zeta)$, where $p=2, \mu=(-3,-9)^{T}, \lambda=$ $(-(1 / 2),-(1 / 2))^{T}$, and $\zeta=(1, \ldots, 1)^{T}$.

We consider the fourth-order explicit method (cf., [17]):

$$
\left\{\begin{array}{l}
Y_{1}^{k}=y_{k, l-1}, \\
Y_{2}^{k}=y_{k, l-1}+\frac{h_{k}}{2} f\left(t_{k, l-1}, Y_{1}^{k}\right)+\frac{h_{k}^{2}}{8} f^{\prime}\left(t_{k, l-1}, Y_{1}^{k}\right), \\
y_{k, l}=y_{k, l-1}+h_{k} f\left(t_{k, l-1}, Y_{1}^{k}\right)+h_{k}^{2}\left[\frac{1}{6} f^{\prime}\left(t_{k, l-1}, Y_{1}^{k}\right)+\frac{1}{3} f^{\prime}\left(t_{k, l-1}+\frac{h_{k}}{2}, Y_{2}^{k}\right)\right] .
\end{array}\right.
$$

Take 


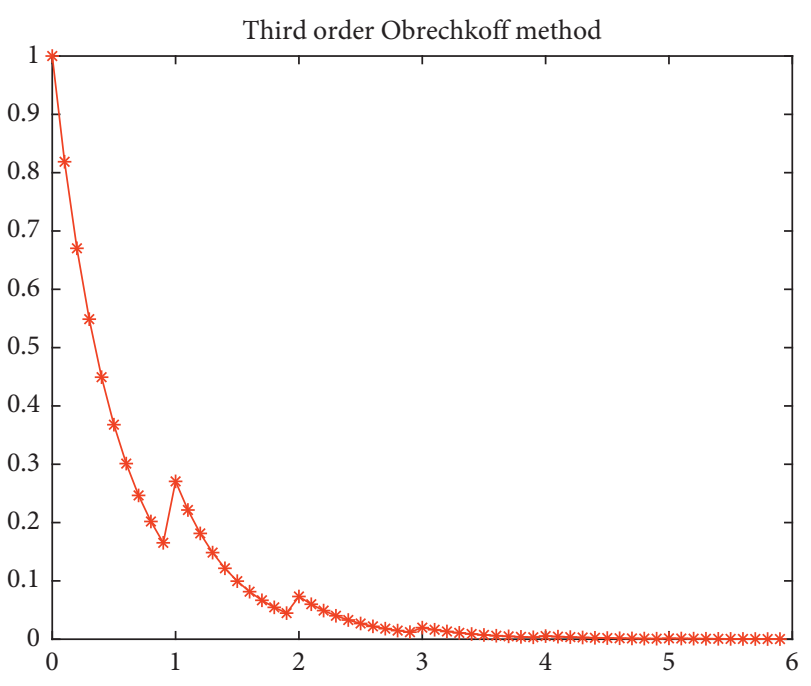

FIGURE 1: The error of $\left\|y_{k, l}-z_{k, l}\right\|_{1}$ for (42) and (43).

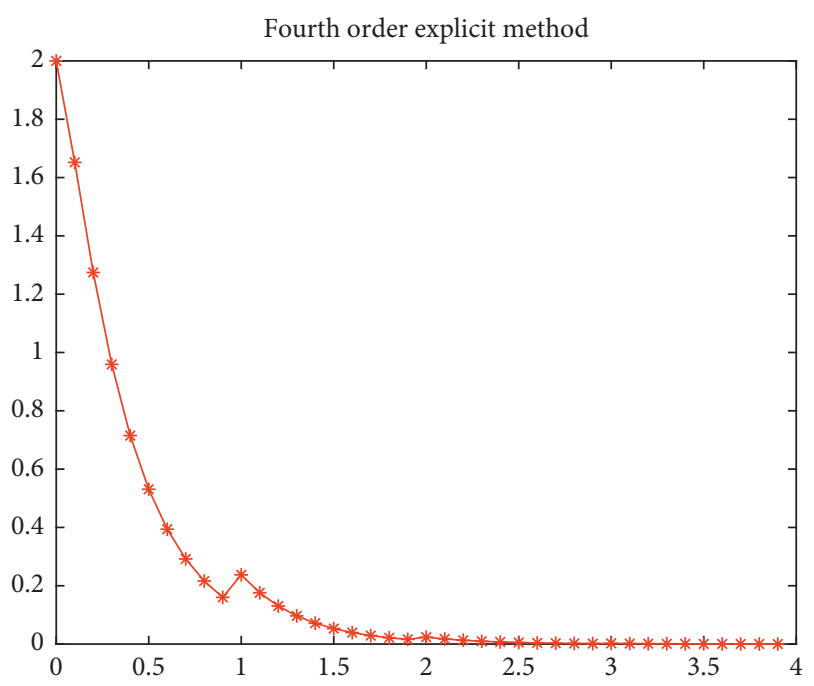

Figure 2: The error of $\left\|y_{k, l}-z_{k, l}\right\|_{1}$ for (48) and (49).

$$
\begin{aligned}
& D^{(1)}=\left(\begin{array}{cc}
\frac{1}{3} & 0 \\
\frac{1}{3} & \frac{1}{6}
\end{array}\right), \\
& D^{(2)}=\left(\begin{array}{cc}
\frac{1}{3} & 0 \\
\frac{1}{6} & \frac{1}{3}
\end{array}\right),
\end{aligned}
$$

which implies that the matrix group

$$
\begin{aligned}
& G^{(1)}=\left(\begin{array}{cc}
-\frac{3}{4} & 0 \\
-\frac{3}{2} & 0
\end{array}\right), \\
& G^{(2)}=\left(\begin{array}{cc}
-\frac{3}{16} & 0 \\
-\frac{5}{24} & -\frac{2}{3}
\end{array}\right) .
\end{aligned}
$$

Thus, we can verify that method (50) is $G_{p}-$ stable. For $\forall h_{k} \in(0, H)$, when method (50) is applied to problems (48) and (49), we have the following stability inequalities:

$$
\begin{array}{r}
\left\|y_{k, l}-z_{k, l}\right\| \leq 2 \times \phi^{l}\left(h_{k}\right) \prod_{i=1}^{k} \phi^{m}\left(h_{i-1}\right)\left\|y_{0}-z_{0}\right\|, \\
l=1,2, \ldots, m,
\end{array}
$$

where $H=\min \left\{-(4 / 3) \lambda_{1}, \sqrt{-(3 / 2) \lambda_{2}}\right\}=\sqrt{(3 / 4)}, \phi\left(h_{k}\right)$ can be obtained by (25).

According to Corollary 2, when we take $h_{k}=(1 / 10)$, the fourth-order explicit method (50) for (48) is asymptotically stable (see Figure 2).

\section{Conclusion}

In this paper, we focus on the stability and asymptotic stability of multistage one-step multiderivative methods for the problem class $K^{(p)}(\mu, \lambda, \zeta)$ in Banach spaces and confirm the correctness of the results through two numerical experiments. In the future work, we might analyze the convergence of multistage one-step multiderivative methods for nonlinear impulsive differential equations.

\section{Data Availability}

The data used to support the results of this study are included in the article.

\section{Conflicts of Interest}

The authors declare that they have no conflicts of interest.

\section{Authors' Contributions}

All authors contributed equally to the writing of this paper. All authors read and approved the final manuscript.

\section{Acknowledgments}

This work was supported by the NSF of China (11 571 291) and Scientific Research Fund of Hunan Provincial Education Department. 


\section{References}

[1] H. J. Guo, L. S. Chen, and X. Y. Song, "Mathematical models of restoration and control of a single species with Allee effect," Applied Mathematical Modelling, vol. 34, no. 11, pp. 32643272, 2016.

[2] M. Qiao, A. Liu, and U. Foryś, "Qualitative analysis of the SICR epidemic model with impulsive vaccinations," Mathematical Methods in the Applied Sciences, vol. 36, no. 6, pp. 695-706, 2013.

[3] Z. Lu, X. Chi, and L. Chen, "Impulsive control strategies in biological control of pesticide," Theoretical Population Biology, vol. 64, no. 1, pp. 39-47, 2003.

[4] C. P. Joseph, D. Jungmann, and S. Siegmund, "A comparative study of integrated pest management strategies based on impulsive control," Journal of Biological Dynamics, vol. 12, no. 1, pp. 318-341, 2018.

[5] Z. Li, X. B. Shu, X.-B. Shu, and F. Xu, "The existence of upper and lower solutions to second order random impulsive differential equation with boundary value problem," AIMS Mathematics, vol. 5, no. 6, pp. 6189-6210, 2020.

[6] Y. C. Guo, X. B. Shu, Y. J. Li, and F. Xu, "The existence and Hyers-Ulam stability of solution for an impulsive RiemannLiouville fractional neutral functional stochastic differential equation with infinite delay of order $1<\beta<2$," Boundary Value Problems, vol. 2019, no. 1, pp. 1-18, 2019.

[7] Q. Wen and J. R. Wang, "Continuous dependence and differentiability of solutions of second-order impulsive differential equations on initial values and impulsive points," Qualitative Theory of Dynamical Systems, vol. 20, no. 3, pp. 1-25, 2021.

[8] L. Wen and Y. Yu, "The analytic and numerical stability of stiff impulsive differential equations in Banach space," Applied Mathematics Letters, vol. 24, no. 10, pp. 1751-1757, 2011.

[9] X. Liu, G. L. Zhang, and M. Z. Liu, "Analytic and numerical exponential asymptotic stability of nonlinear impulsive differential equations," Applied Numerical Mathematics, vol. 81, pp. 40-49, 2014.

[10] G. L. Zhang and M. H. Song, "Asymptotical stability of Runge-Kutta methods for advanced linear impulsive differential equations with piecewise constant arguments," Applied Mathematics and Computation, vol. 259, pp. 831-837, 2015.

[11] Y. X. Yu, H. Y. Wen, and R. Xiao, "Stability and convergence of Runge-Kutta methods for stiff impulsive differentiaal equations," Journal of Xiangtan University (Natural Science Edition), vol. 42, pp. 1-6, 2016, in Chinese.

[12] L. Mei, "A novel method for nonlinear impulsive differential equations in broken reproducing kernel space," Acta Mathematica Scientia, vol. 40, no. 3, pp. 723-733, 2020.

[13] G. L. Zhang, "Asymptotical stability of Runge-Kutta methods for nonlinear impulsive differential equations," Advances in Difference Equations, vol. 2020, no. 1, pp. 1-12, 2020.

[14] S. F. Li and B. Ruan, "Nonlinear stability of multistep multiderivative methods," Mathematics of Computation, vol. 55, no. 192, pp. 581-589, 1990.

[15] D. Goeken and O. Johnson, "Runge-Kutta with higher order derivative approximations," Applied Numerical Mathematics, vol. 34, no. 2-3, pp. 207-218, 2000.

[16] D. Goeken and O. Johnson, "Fifth-order Runge-Kutta with higher order derivative approximations," The Electronic Journal of Differential Equations, vol. 2, pp. 1-9, 1999.

[17] E. Gekeler and R. Widmann, "On the order conditions of Runge-Kutta methods with higher derivatives," Numerische Mathematik, vol. 50, no. 2, pp. 183-203, 1986.
[18] C. J. Zhang, "The nonlinear stability of a class of multistage one-step multiderivative methods," Mathematica Numerica Sinica, vol. 18, pp. 46-53, 1996, in Chinese.

[19] D. Bainov and P. Simeonov, Systems with Impulsive Effect: Stability, Theory and Applications, Ellis Horwood, Chichester, UK, 1989.

[20] G. L. Zhang, "Asymptotical stability of numerical methods for semi-linear impulsive differential equations," Computational and Applied Mathematics, vol. 39, no. 1, pp. 1-21, 2020. 